\title{
Dispersion relation of Al/Si surface plasmon in hexagonally ordered aluminum hole arrays
}

\author{
Yi-Tsung Chang, Tzu-Hung Chuang, Ming-Wei Tsai, Lung-Chien Chen, and Si-Chen Lee ${ }^{a}$ \\ Department of Electrical Engineering, Graduate Institute of Electronics Engineering, National Taiwan \\ University, Taipei, Taiwan 106, Republic of China
}

(Received 20 September 2006; accepted 7 December 2006; published online 5 March 2007)

\begin{abstract}
This investigation examines the extraordinary light transmission through periodic hexagonally ordered aluminum hole arrays on silicon substrates. The holes are circular and the arrays with various hole diameters were fabricated. The transmission spectra through the hole arrays were measured as a function of the incident angle of the light beam. It is observed that the transmission peak splits into two when the diameter of the hole is close to half of the lattice constant. At larger incident angles, the six degenerate $(1,0) \mathrm{Al} / \mathrm{Si}$ surface plasmon modes split into four or three modes depending on the symmetry axis, and even higher order modes can be identified for small hole diameters. () 2007 American Institute of Physics. [DOI: 10.1063/1.2435069]
\end{abstract}

\section{INTRODUCTION}

Numerous mechanisms have been proposed recently to explain extraordinary transmission through subwavelength holes since its discovery in 1998 by Ebbesen et al. ${ }^{1}$ Surface plasmons (SPs) are widely accepted to be involved in the main mechanism that underlies the observed phenomenon. ${ }^{1-3}$ SPs have been utilized in the field of magneto-optic data storage, ${ }^{4,5}$ microscopy, ${ }^{6}$ and solar cells, ${ }^{7}$ and have been adopted to construct sensors to detect biologically interesting molecules. ${ }^{8}$ These authors revealed that the periodic metal hole arrays exhibited enhanced extraordinary light transmission. The phenomenon has also been called as enhanced or resonant transmission, with a periodic metal hole array that substantially exceeds the hole-surface-to-total-surface ratio, exhibiting similarities to the transmission behavior of frequency-selective surfaces, when the wavelength of the incident radiation exceeds the periodicity of the hole arrays. The theory of extraordinary transmission ${ }^{9,10}$ has elucidated the relationship between the periodicity of the hole arrays, the thickness of the film, and the type of metal of which the hole arrays are manufactured. Experiments and calculations have established that the shape and size of holes affect the polarization characteristics of the transmission, ${ }^{11}$ but highorder modes of aluminum on silicon $(\mathrm{Al} / \mathrm{Si})$ surface plasmon have not yet been analyzed.

Therefore, the extraordinary transmission spectra and the dispersion relations of the $\mathrm{Al} / \mathrm{Si}$ surface plasmon polariton (SPP) through aluminum hexagonal-ordered hole arrays were obtained and investigated herein.

\section{THEORY OF SURFACE PLASMONS}

Surface plasmons, comprising electromagnetic waves and surface charge at the interface between a metal and a dielectric material, exhibit the combined characteristics of both materials. Controlling the SPs by adjusting the material and structural parameters, such as the periodicity of the

${ }^{a)}$ Electronic mail: sclee@cc.ee.ntu.edu.tw structure, and the dielectric properties of the interface is important, such that a SP resonant coupling condition yields propagation in a given direction. For a metal-dielectric interface, solving Maxwell's equations under suitable boundary conditions yields the SP dispersion relation. Furthermore the frequency-dependent SP wave vector $\mathbf{k}_{\mathrm{sp}}$ is given by

$$
\left|\mathbf{k}_{\mathrm{sp}}\right|=\frac{\omega}{c} \sqrt{\frac{\varepsilon_{d} \varepsilon_{m}}{\varepsilon_{d}+\varepsilon_{m}}},
$$

where $\varepsilon_{m}$ and $\varepsilon_{d}$ are the frequency-dependent relative dielectric constants of the metal and the dielectric material, respectively, $\omega$ is the frequency of the SP which equals that of the incident radiation, and $c$ is the velocity of light. The electromagnetic field perpendicular to the interface is said to be evanescent, reflecting the bound, nonradiative nature of SPs, and it prevents power from propagating away from the surface. In the dielectric medium above the metal, the decay length of the field $\delta_{d}$ is of the order of half the wavelength of the light involved, whereas the decay length into the metal $\delta_{m}$ is determined by the skin depth ( $\mathrm{Al} \delta_{m}=18.4 \mathrm{~nm}$ at a wavelength of approximately $14.7 \mu \mathrm{m}$ ).

As periodic texturing of the metal surface can cause the formation of a SP photonic band gap when the diameter of the hole is close to half of the periodicity of the metal film, two SP standing waves with frequencies $\omega_{+}$and $\omega_{-}$are formed with different energies. Each mode has its own field and surface charge distribution, causing peak separation in the dielectric/metal interface, as shown in Fig. 1(a). The higher frequency solution $\omega_{+}$is associated with the greater distance between the positive and the negative surface charges and the greater distortion of the field, as presented in Fig. 1(b). The frequencies between the two band edges $\omega_{+}$ and $\omega_{-}$are known as a stop band. At the band edges, the density of SP states is high, and the associated field enhancement is significantly increased. ${ }^{12}$ Figure 1 (c) displays an example of a hexagonal-ordered hole array, in which the lattice constant a is $5 \mu \mathrm{m}$, the radii of hole $r$ are $0.75,1$, and $2 \mu \mathrm{m}$, and the $\mathrm{Al} \mathrm{film}$ is $300 \mathrm{~nm}$ in thickness. The transmission 


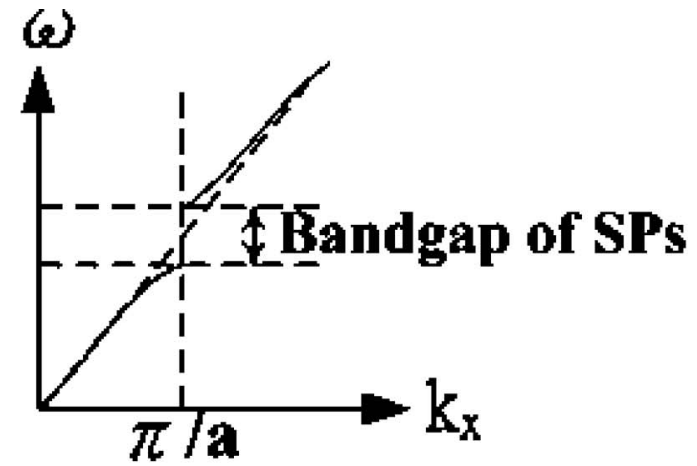

(a)

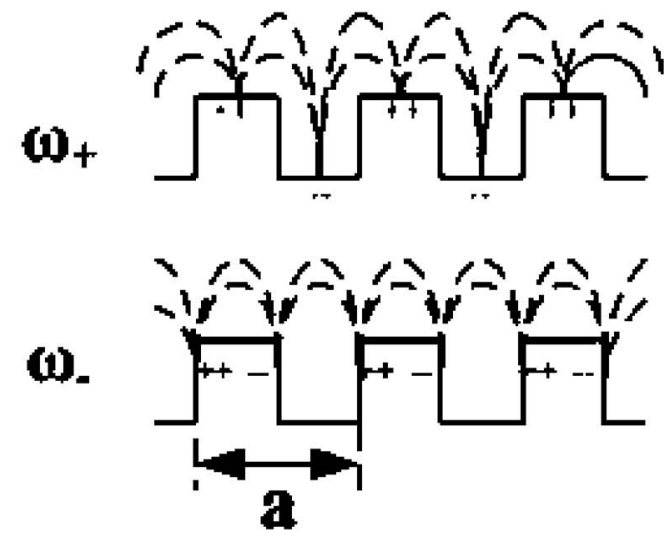

(b)

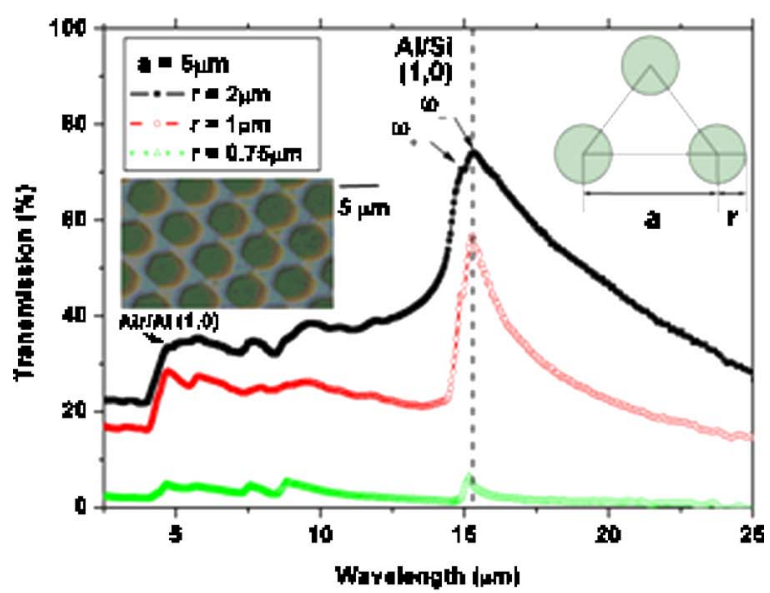

(c)

FIG. 1. (Color online) (a) Dispersion relation of a plasmon on a metal surface with periodic hole array. (b) Charge distribution of $\omega_{+}$and $\omega_{-}$modes in a two-dimensional periodic hole array. (c) For a hexagonal-ordered hole array $(a=5 \mu \mathrm{m}, r=0.75,1$, and $2 \mu \mathrm{m}$, and Al film thickness $t=300 \mathrm{~nm}$ ), the broad transmission peak comprises two peaks because of the Bragg diffraction.

peak consists of two peaks, the smaller of which on the left side at a wavelength of approximately $15 \mu \mathrm{m}$ is the $\omega_{+}$ mode, and the larger of which on the right side at a wavelength of $15.3 \mu \mathrm{m}$ is the $\omega_{-}$mode. As the size of the holes increases and the metal linewidth falls, the $\omega_{-}$mode that involves the formation of a charge dipole in the metal becomes more and more difficult, reducing the amplitude.

If a two-dimensional (2D) periodic array of apertures is introduced, the energy and momentum are conserved if

$$
\mathbf{k}_{\mathrm{sp}}=\mathbf{k}_{x} \pm m \mathbf{G}_{x} \pm n \mathbf{G}_{y},
$$

where $\mathbf{k}_{x}$ is the wave vector of the incident light in the $x$ direction $\left(\left|\mathbf{k}_{x}\right|=(2 \pi / \lambda) \sin \Omega=0.83 \times 10^{6} \mathrm{~m}^{-1}\right.$ at an incident angle $\Omega=30^{\circ}$ and the wavelength of the incident light $\lambda$ $=15.2 \mu \mathrm{m})$, and $\mathbf{G}_{X}=(4 \pi / \sqrt{3} a)[(1 / 2) \mathbf{x}+(\sqrt{3} / 2) \mathbf{y}]$ and $\mathbf{G}_{y}$ $=(4 \pi / \sqrt{3} a)[(1 / 2) \mathbf{x}-(\sqrt{3} / 2) \mathbf{y}]$ are the reciprocal lattice vectors associated with the hexagonal array. " $m$ " and " $n$ " are integers that represent the order of the scattering event that couples incident light with a SP mode with wave vector $\mathbf{k}_{\mathrm{sp}}$. At normal incidence, the transmission spectra of the periodic array of holes exhibits peaks that can be tuned by adjusting the period and the symmetry of the array. For a hexagonal hole array with period $a$, the peak transmission wavelength

$$
\lambda_{\max }=a\left[\frac{4}{3}\left(m^{2}-m n+n^{2}\right)\right]^{-1 / 2}\left(\frac{\varepsilon_{d} \varepsilon_{m}}{\varepsilon_{d}+\varepsilon_{m}}\right)^{1 / 2}
$$

can be determined approximately from the dispersion relation Eqs. (1) and (2). The prediction of Eq. (3) is highly consistent with the measured positions of the SP resonances for various values of $m$ and $n \cdot{ }^{13-15}$ Notably, the air/metal and metal/dielectric interfaces each contribute a set of $(m, n)$ maxima that are distinguishable because the real parts of the dielectric constants of air $\left(\varepsilon_{\text {air }}=1\right)$, the metal $\left(\varepsilon_{\mathrm{Al}}=-1.69\right.$ $\times 10^{4}$ at wavelength of $16 \mu \mathrm{m}$ ), and the dielectric material (Si wafer, $\varepsilon_{\mathrm{Si}}=11.9$ ) vary significantly. ${ }^{16}$ Both peaks are observed in the transmission spectra. Varying the incident angle of the radiation yields the dispersion relation of the SPP. ${ }^{17-23}$

\section{EXPERIMENTS}

The metal films examined in this study were prepared by depositing a $300 \mathrm{~nm}$ thick aluminum $(\mathrm{Al})$ layer on the doubly polished $p$-type silicon wafer, and the photoresist was spun onto $\mathrm{Al}$ films for lithography. Following the pattern transfer, the hexagonal-ordered circular hole arrays were fabricated by wet etching. The lattice constant was in the range 3-6 $\mu \mathrm{m}$. The radius of the holes was between 0.5 and $2 \mu \mathrm{m}$. The Al metal film was perforated over an area of $1 \times 1 \mathrm{~cm}^{2}$.

Then, the transmission spectra were obtained using a Bruker IFS 66V/S Fourier-transform infrared (FTIR) spectrometer. The zero-order transmission spectra (for which the detected beam emerges collinear with the incident beam) were obtained using light that was normally incident onto the surface of the Al film with perforated holes, and the symmetry of the optical spectra in hexagonal-ordered aluminum hole array transmission spectra was analyzed. The SP modes and band structure obtained as the incident angle $\Omega$ of the radiation is varied were considered, as shown in Figs. 2 and 3 , respectively. The wave number resolution of the transmission spectra was $8 \mathrm{~cm}^{-1}$; the incident light was in the $z$ direction; the sample was defined to lie in the $(x, y)$ plane, and it was separately rotated around the $x$ and $y$ axes.

\section{RESULTS AND DISCUSSION}

\section{A. Symmetry of optical spectra in hexagonal-ordered aluminum hole arrays}

Figure 2 displays the transmission spectra of the hexagonal-ordered circular hole array (lattice constant $a$ 


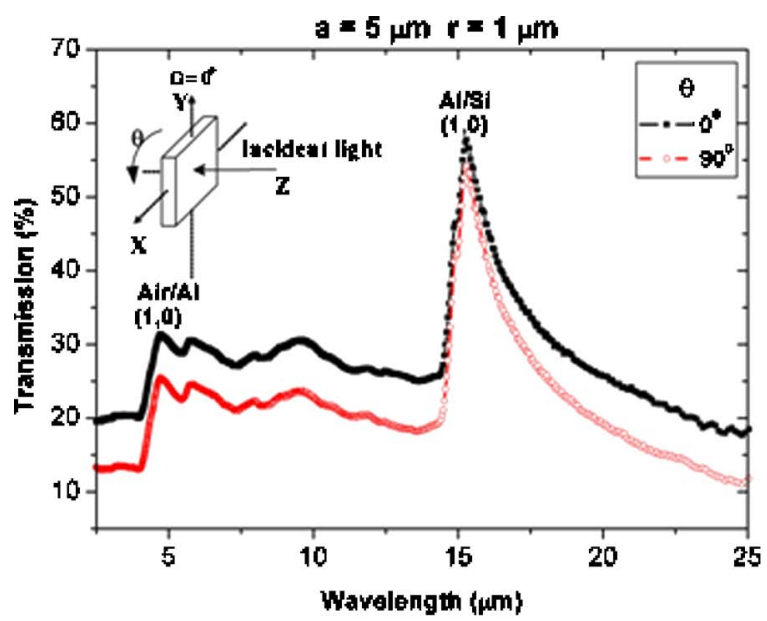

(a)

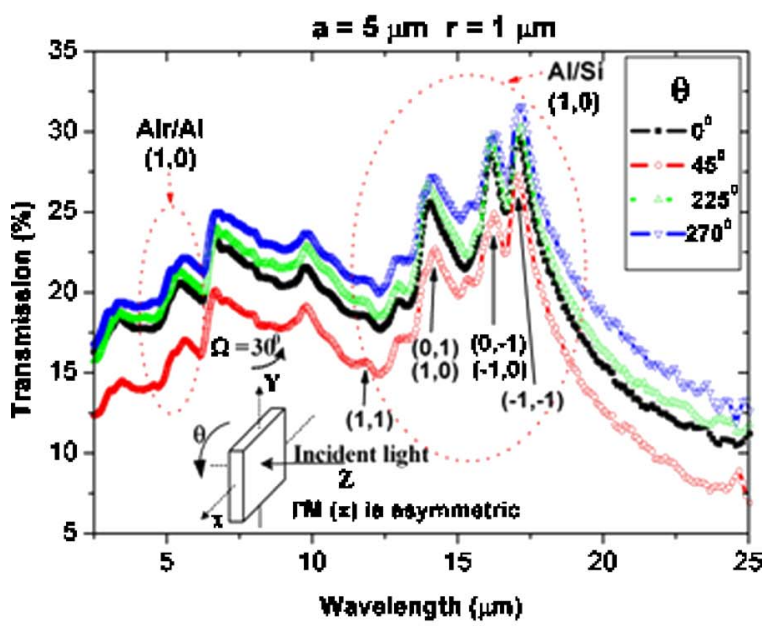

(b)

FIG. 2. (Color online) Transmission spectra obtained by rotating the $\mathrm{Al} / \mathrm{Si}$ hexagonal hole array $a=5 \mu \mathrm{m}$ and $r=1 \mu \mathrm{m}$ (a) relative to the incident light by rotating the sample by $0^{\circ}$ and $90^{\circ}$ around the $z$ axis, as displayed in the inset; (b) around the $y$ axis by $30^{\circ}$ and then by $0^{\circ}, 45^{\circ}, 225^{\circ}$, and $275^{\circ}$ around the $z$ axis, as displayed in the inset.

$=5 \mu \mathrm{m}$, hole radius $r=1 \mu \mathrm{m}$, and Al film thickness $t$ $=300 \mathrm{~nm}$ ). The inset in Fig. 2(a) presents the experimental setup. First, the sample was immobile in the $x-y$ plane $(\Omega$ $=0^{\circ}$ ); the incident light was along the $z$ direction, and the transmission spectra were obtained by rotating the sample around the $z$ axis, from $\theta=0^{\circ}-90^{\circ}$. The transmission intensity varies because of the symmetry of the hexagonal hole pattern. The two peak transmission wavelengths at 4.4 and $15.2 \mu \mathrm{m}$ satisfy Eq. (3) and correspond to air/ $\mathrm{Al}$ and $\mathrm{Al} / \mathrm{Si}$ $(1,0)$ plasmon modes, respectively. Similarly, in the inset of Fig. 2(b), first, the sample was rotated counterclockwise by $30^{\circ}\left(\Omega=30^{\circ}\right)$ around the $y$ axis; then the transmission spectra were obtained by rotating the sample around the $z$ axis through $\theta=0^{\circ}, 45^{\circ}, 225^{\circ}$, and $270^{\circ}$. Only the transmission intensities but not the peak wavelengths varied, indicating that the symmetry of this structure was extremely good.

\section{B. Effect of hole size on the transmission spectra and dispersion relation}

Samples in which $300 \mathrm{~nm}$ thick Al films were deposited on doubly polished $p$-Si wafers were fabricated to elucidate the dependence of the transmission spectra on the size of the holes. The transmission spectra were measured in a series of samples with a period of $a=5 \mu \mathrm{m}$ and hole radii of $r=2,1$, and $0.75 \mu \mathrm{m}$, and the transmission peak positions are in association with incident light coupled to SP modes. First, the sample was immobile in the $x-y$ plane $\left(\theta=0^{\circ}\right)$, the transmission spectra of a hexagonal-ordered hole array were measured by rotating the sample counterclockwise around the $y$ axis as displayed in the insets in Figs. 3(a), 3(c), and 3(e), in which $\Omega$ is increased in steps of $1^{\circ}-50^{\circ}$, and according to Eqs. (2) and (3), the gray-scale dispersion relation of SPP is as presented in Figs. 3(b), 3(d), and 3(f). The inset in Fig. 3(a) displays the geometry of the sample and the measurement scheme. The hole radius is $2 \mu \mathrm{m}$. At longer wavelengths, the spectrum in Fig. 3(a) is characterized by a set of maxima whose positions are governed by the geometry of the array and Eq. (3), which will be discussed below. At the maxima, a surprisingly large transmission is observed-in this case $T \sim 75 \%$. Since the holes cover $\sim 58 \%$ of the total area of the $\mathrm{Al}$ metal film, the results are independent of whether the illumination impinges from the $\mathrm{Al}$ or $\mathrm{Si}$ side. The six SP modes $[(-1,-1),(1,1),(1,0),(0,1),(0,-1)$, and $(-1,0)]$ identified as the $(1,0) \mathrm{Al} / \mathrm{Si}$ mode are degenerate at normal incidence, as shown in Fig. 3(b). SPs can be excited in a given direction by appropriately selecting the wavelength $\lambda$ and the angle $\Omega$ of the incident light, based on Eq. (2). ${ }^{24}$ According to Eq. (3), the six degenerate $(1,0) \mathrm{Al} / \mathrm{Si}$ SP modes at normal incidence of radiation will be at a wavelength of approximately $15.0 \mu \mathrm{m}$; the measured value is $15.2 \mu \mathrm{m}$, indicating a slight redshift from the theoretical value. When the incident radiation is tilted toward the $x$ axis, $k_{x}$ is no longer zero; the forward SPP wave and the backward SPP wave interfere with each other, and the electric field polarized in the $y$ direction exceeds that polarized in the $x$ direction. $(-1,-1)$ and $(-1,0) /(0,-1) \mathrm{Al} / \mathrm{Si}$ modes split at $\Omega=20^{\circ}$ and redshift to longer wavelengths and lower energy, whereas $(1,0) /(0,1)$ and $(1,1) \mathrm{Al} / \mathrm{Si}$ modes blueshift to shorter wavelengths. In Fig. 3(a), when the incident angle $\Omega=0^{\circ}$, the spilt double peaks represent degenerate modes that comprise $(1,1)$ and $(-1,-1) \mathrm{Al} / \mathrm{Si}$ modes, because the photonic band gap opens up when the size of the hexagonalordered hole exceeds half of the lattice constant $a / 2$ and the normally incident light propagates through the sample of periodic perforated hexagonal-ordered hole arrays, yielding two standing wave solutions with their own field and surface charge distributions. In Fig. 3(a), the $(1,-1)$ and $(2,-1)$ $\mathrm{Al} / \mathrm{Si}$ modes appeared at wavelengths of approximately 8.6 and $5.6 \mu \mathrm{m}$, respectively, which are very close to theoretical values predicted by Eq. (3). Since the intensities of the two SP modes are small, plotting the dispersion relation in Fig. 3 (b) is difficult. As the incident angle $\Omega$ increases to $30^{\circ}$, the $(2,0) \mathrm{Al} / \mathrm{Si}$ mode appears. Also, according to Eq. (3), since $\varepsilon_{\text {air }}=1$, the $(1,0)$ air/Al SP mode is at a wavelength of approximately $4.4 \mu \mathrm{m}$. It is difficult to analyze because the transmission intensity is small.

Figure 3(c) presents the transmission spectra of hexagonal-ordered hole arrays with $a=5 \mu \mathrm{m}$ and radius $r$ $=1 \mu \mathrm{m}$ on the $\mathrm{Al}$ film by rotating the sample around the $y$ axis, with $\theta$ fixed at $0^{\circ}$. Since the holes cover $\sim 14.5 \%$ of the 


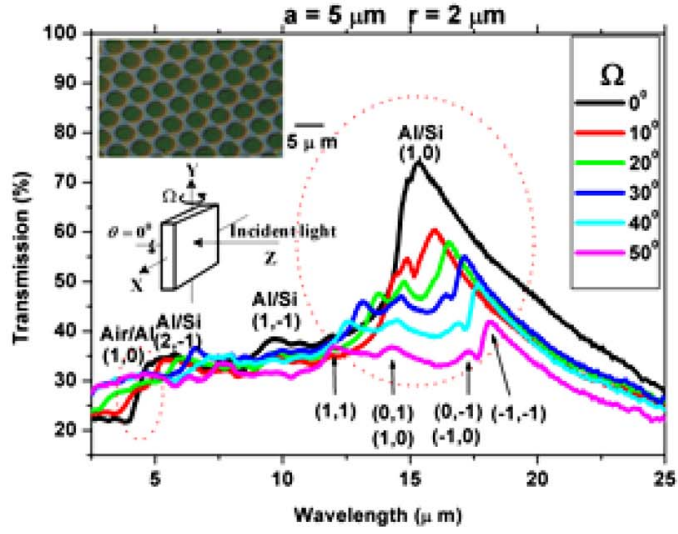

(a)

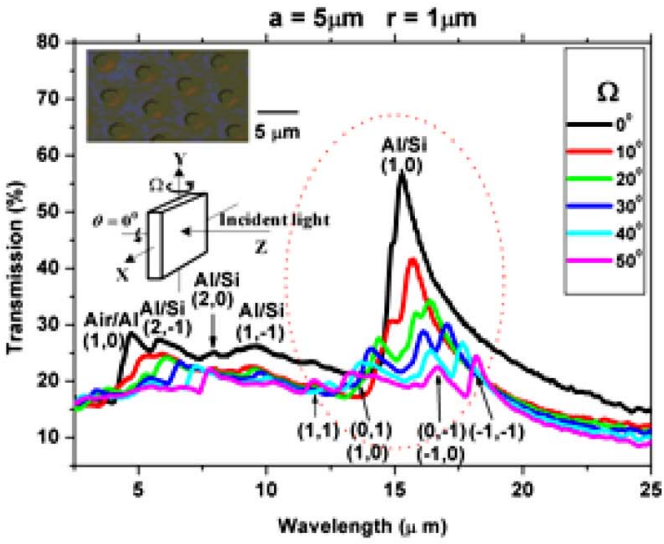

(c)

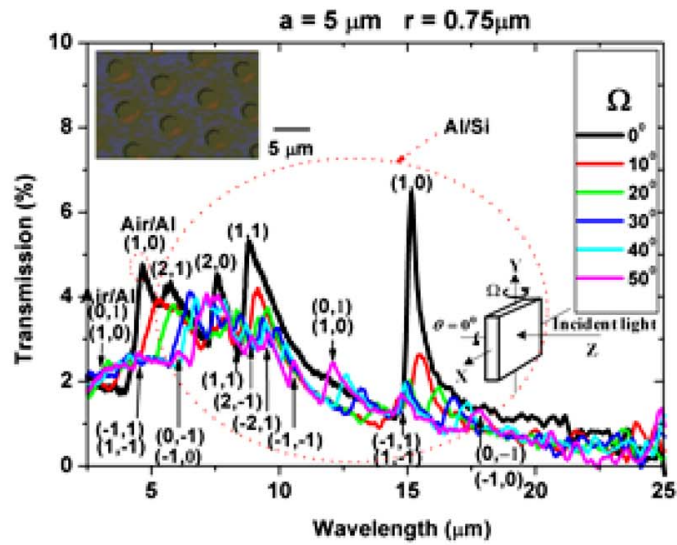

(e)

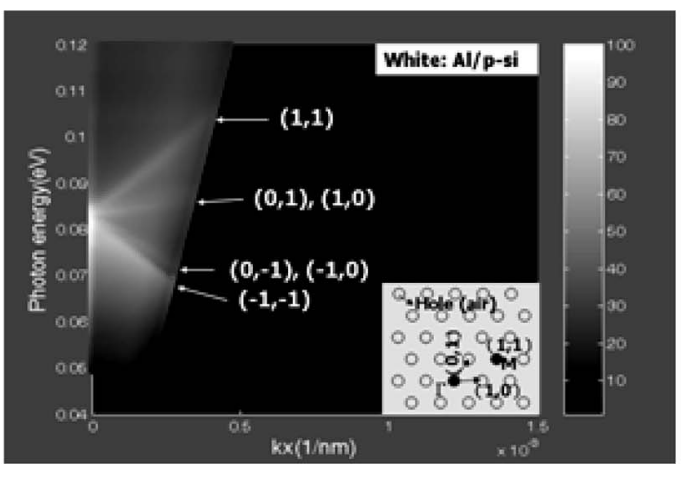

(b)

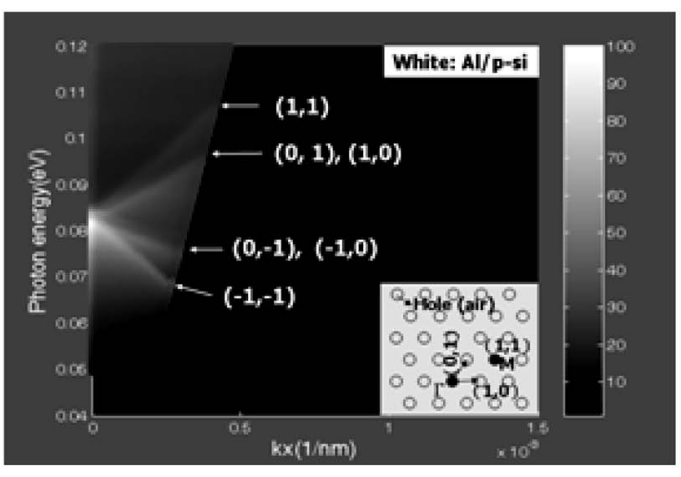

(d)

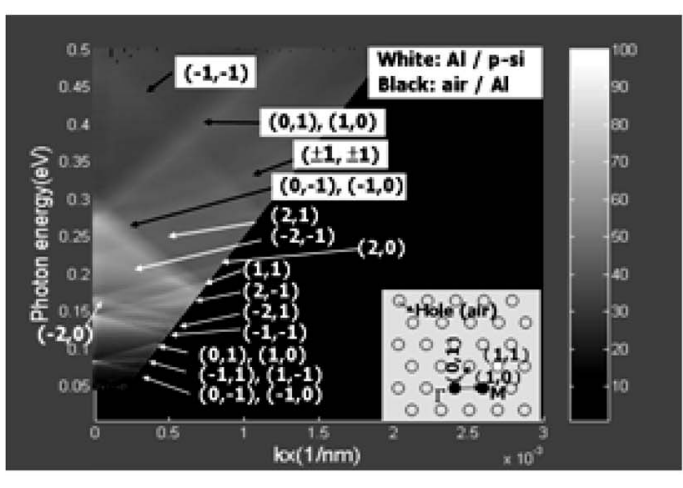

(f)

FIG. 3. (Color online) Zero-order transmission spectra and dispersion relations obtained by rotating the $\mathrm{Al} / \mathrm{Si}$ sample around the $y$ axis. The spectra and dispersion relations were obtained from $1^{\circ}$ increments to $50^{\circ}$ for hexagonal-ordered aluminum hole arrays relative to the incident light, periodicity of sample $a=5 \mu \mathrm{m}$, and thickness $t=300 \mathrm{~nm}$. (a) Spectra and (b) dispersion relation for the sample $r=2 \mu \mathrm{m}$ (white: Al/Si modes). (c) Spectra and (d) dispersion relation for the sample $r=1 \mu \mathrm{m}$ (white: Al/Si modes). (e) Spectra and (f) dispersion relation for the sample $r=0.75 \mu \mathrm{m}$ (white: Al/Si modes, green: air/Al modes).

total area of the $\mathrm{Al}$ metal film, the maximum transmission at $15.2 \mu \mathrm{m}$ declines to $T \sim 58 \%$. The six modes $[(-1,-1)$, $(1,1),(1,0),(0,1),(0,-1)$, and $(-1,0)]$ identified as the $(1,0) \mathrm{Al} / \mathrm{Si}$ mode are also degenerate, as displayed in Fig. 3(d). According to Eq. (3), the short wavelength transmission peak $(\sim 4.4 \mu \mathrm{m})$ is associated with $(1,0)$ air/Al SP modes, corresponding to an energy of $0.28 \mathrm{eV}$. The transmission in- tensity of the $(1,-1)$ and $(2,-1) \mathrm{Al} / \mathrm{Si}$ modes are clearly visible, but the degenerate modes still cannot be analyzed.

Figure 3(e) displays the transmission spectrum of a hexagonal Al hole array with lattice $a=5 \mu \mathrm{m}$ and radius $r$ $=0.75 \mu \mathrm{m}$ on the Al film. Since the holes cover only $\sim 8 \%$ of the total area of the $\mathrm{Al}$ metal film, the maximum transmission efficiency falls to $T \sim 7 \%$. The sample was rotated 
around the $z$ axis for $30^{\circ}$, therefore, the six $(1,0) \mathrm{Al} / \mathrm{Si}$ modes which are degenerate at normal incidence split into three modes instead of four modes shown in Figs. 3(b) and 3(d) when the incident angle between the light beam and substrate increases along the $x$ axis. In the transmission peak $(\sim 8.8 \mu \mathrm{m})$, the four SP modes $[(1,1),(2,-1),(-2,1)$, and $(-1,-1)]$ identified as $(1,1) \mathrm{Al} / \mathrm{Si}$ mode are also degenerate at normal incidence, as shown in Fig. 3(e). The $(1,0),(1,1)$, and $(2,1)$ SP modes are clearly visible, and are compared with Fig. 3(c). The $(2,0),(-2,0),(2,1)$, and $(-2,-1)$ SP modes identified as $(2,0)$ and $(2,1) \mathrm{Al} / \mathrm{Si}$ modes are also degenerate, as presented in Figs. 3(e) and 3(f). The four $\mathrm{Al} / \mathrm{Si} \mathrm{SP}$ high-order modes $[(1,1),(2,0),(2,1)$, and $(2,2)]$ are analyzed and presented in Figs. 3(e) and 3(f). Similarly analyzing the $(1,0) \mathrm{Al} / \mathrm{Si} \mathrm{SP}$ mode demonstrates that the six $(1,0)$ air/Al modes are also degenerate at normal incidence, but the $(-1,1)$ and $(-1,0)$ air/Al SP modes hide in the $(2,1)$ and $(2,0) \mathrm{Al} / \mathrm{Si} \mathrm{SP}$ modes, as presented in Figs. 3(e) and $3(\mathrm{f})$.

\section{CONCLUSIONS}

The transmission peak splits into two when the diameter of the air hole is close to half of the lattice constant. The symmetry of the fabricated hexagonal structure is extremely good as the sample is rotated around the incident light with transmission peak positions. The plasmon dispersion relations - especially those of the higher order modes-are established. As the hole size becomes smaller, higher order SP modes tend to appear, thus yielding the complete dispersion relation between $\mathrm{Al}$ and $\mathrm{Si}$ surface plasmon polaritons.

\section{ACKNOWLEDGMENTS}

The authors would like to thank the National Science Council of the Republic of China, Taiwan, for financially supporting this research under Contract No. NSC 94-2120M-002-013.
${ }^{1}$ T. W. Ebbesen, H. J. Lezec, H. F. Ghaemi, T. Thio, and P. A. Wolff, Nature (London) 391, 667 (1998).

${ }^{2}$ W. L. Barnes, A. Dereux, and T. W. Ebbesen, Nature (London) 424, 824 (2003).

${ }^{3}$ K. L. van der Molen, K. J. Klein KoerKamp, S. Enoch, F. B. Segerink, N. F. van Hulst, and L. Kuipers, Phys. Rev. B 72, 045421-1 (2005).

${ }^{4}$ E. Betzig, J. K. Trautman, R. Wolfe, E. M. Gyorgy, P. L. Finn, M. H. Kryder, and C.-H. Chang, Appl. Phys. Lett. 61, 142 (1992).

${ }^{5}$ L. P. Shi, T. C. Chong, H. B. Yao, P. K. Tan, and X. S. Miao, J. Appl. Phys. 91, 10209 (2002).

${ }^{6}$ U. Schroter and A. Dereux, Phys. Rev. Lett. 64, 125420 (2001).

${ }^{7}$ N. M. Lawandy, Proc. SPIE 5924, 59240G-1 (2005).

${ }^{8}$ K. Kneipp, H. Kneipp, I. Itzkan, R. R. Dasari, and M. S. Feld, J. Phys.: Condens. Matter 14, R597 (2002).

${ }^{9}$ L. Martín-Moreno, F. J. García-Vidal, H. J. Lezec, K. M. Pellerin, T. Thio, J. B. Pendry, and T. W. Ebbesen, Phys. Rev. Lett. 86, 1114 (2001).

${ }^{10}$ S. A. Darmanyan and A. V. Zayats, Phys. Rev. B 67, 035424 (2003).

${ }^{11}$ K. L. van der Molen, F. B. Segerink, and N. F. van Hulst, Appl. Phys. Lett. 85, 4316 (2004).

${ }^{12}$ A. Giannattasio and W. L. Barnes, Opt. Express 13, 428 (2005).

${ }^{13}$ D. W. Lynch and W. R. Hunter, in Handbook of Optical Constants of Solids, edited by E. D. Palik (Academic, Orlando, 1985).

${ }^{14}$ T. Thio, H. F. Ghaemi, H. J. Lezec, P. A. Wolff, and T. W. Ebbesen, J. Opt. Soc. Am. B 16, 1743 (1999).

${ }^{15}$ W. L. Barnes, W. A. Murray, J. Dintinger, E. Devaux, and T. W. Ebbesen, Appl. Phys. Lett. 92, 107401-1 (2004).

${ }^{16}$ M. A. Ordal, L. L. Long, R. J. Bell, S. E. Bell, R. R. Bell, R. W. Alexander, Jr., and C. A. Ward, Appl. Opt. 22, 1099 (1983).

${ }^{17}$ H. A. Bethe, Phys. Rev. 66, 163 (1944).

${ }^{18}$ H. F. Ghaemi, T. Thio, D. E. Groupp, T. W. Ebbesen, and H. J. Lezec, Phys. Rev. B 58, 6779 (1998).

${ }^{19}$ L. Martin-Moreno, F. J. Garcia-Vidal, H. J. Lezec, K. M. Pellerin, T. Thio, J. B. Pendry, and T. W. Ebbesen, Phys. Rev. Lett. 86, 1114 (2001).

${ }^{20}$ J. Elliott, I. I. Smolyaninov, N. I. Zheludev, and A. V. Zayats, Phys. Rev. B 70, 233403-1 (1998).

${ }^{21}$ J. Elliott, I. I. Smolyaninov, N. I. Zheludev, and A. V. Zayats, Opt. Lett. 29, 1414 (2004)

${ }^{22}$ R. Gordon, A. G. Brolo, A. McKinnon, A. Rajora, B. Leathem, and K. L. Kavanagh, Phys. Rev. Lett. 92, 037401-1 (2004).

${ }^{23}$ K. J. K. Koerkamp, S. Enoch, F. B. Segerink, N. F. Van Hulst, and L. Kuipers, Phys. Rev. Lett. 92, 183901-1 (2004).

${ }^{24}$ D. Eforov, B. S. Dennis, G. Blumberg, and M. I. Haftel, Phys. Rev. B 70, 033404-1 (2004). 